\title{
¿Son culpables los altos mandos de promover ejecuciones extrajudiciales en Colombia?
}

\section{Are the high-ranking officials guilty of promoting extrajudicial executions in Colombia?}

\section{Sebastián Durán",María Castillo Valencia"}

\author{
Universidad del Valle
}

Recibido: 23 de marzo de 2020-Aceptado: 31 de mayo de 2021-Publicado: 1 de enero de 2022

Forma de citar este artículo en APA:

Duran, S., \& Castillo-Valencia, M. (2022). ¿Son culpables los altos mandos de promover ejecuciones extrajudiciales en Colombia? Revista Colombiana de Ciencias Sociales, 13(1), 43-76. https://doi.org/10.21501/22161201.3574

\section{Resumen}

El objetivo de este trabajo es estudiar la naturaleza de las ejecuciones extrajudiciales en Colombia, teniendo como hipótesis que estas acciones no fueron hechos aislados, sino que, por el contrario, pudieron ser ordenadas por los altos mandos militares. La revisión de literatura y la evidencia obtenida de los relatos y denuncias de sus protagonistas muestran que estos hechos ocurrieron bajo la dirección de algunos mandos medios y superiores. Usando el enfoque teórico del modelo de principal-agente, desarrollado por DeMeritt (2015), se muestra que la impunidad de la que gozaban los oficiales de alto rango del Ejército colombiano

Economista, Universidad del Valle. Contacto: sebastian.duran@correounivalle.edu.co

“" Ph. D. en Economía de la Universidad Federal de Rio Grande do Sul, Brasil. Profesora de Economía y directora del Grupo Conflicto, Aprendizaje y Teoría de Juegos, Facultad de Ciencias Sociales y Económicas, Universidad del Valle, Cali-Colombia. Contacto: maria.d.castillo@correounivalle.edu.co. ORCID: https://orcid.org/0000-0003-4228-3902, Google Scholar: https:/l scholar.google. com/citations?hl=en\&user=PDmDOiQAAAAJEview_op=list_works\&sortby=pubdate 
llevó a la comisión de estos crímenes de manera sistemática. Por tanto, su solución no se remite tan solo al control -ya que gran parte de la estructura militar estaba involucrada-, sino a la reestructuración de la política que mide el éxito de la unidad militar mediante el número de bajas humanas.

\section{Palabras clave}

Colombia; Falsos positivos; Modelo principal agente; Teoría de juegos; Ejército.

\section{Abstract}

The objective of this work is to study the nature of extrajudicial executions in Colombia, having as a hypothesis that these actions were not isolated events, but, on the contrary, could be ordered by the high military commands. The literature review and the evidence obtained from the stories and denunciations of its protagonists show that these events occurred under the direction of some middle and senior managers. Using the theoretical approach of the principal-agent model developed by Demeritt (2015), it is shown that the impunity enjoyed by high-ranking officers of the Colombian army led to the commission of these crimes in a systematic manner. Therefore, its solution does not refer only to control, since much of the military structure was involved, but to the restructuring of the policy that measures the success of the military unit employing the number of casualties.

\section{Keywords}

Colombia; False positives; Principal-agent model; Game theory; Army. 


\section{Introducción}

La sombra de los falsos positivos no deja de perseguir a Colombia. En febrero del 2021, la JEP ${ }^{1}$ habla de que al menos 6402 personas fueron muertas ilegítimamente entre el 2002 y 2008 para ser presentadas como bajas en combate en todo el territorio nacional (JEP, 18 de febrero del 2021). En un medio internacional aparece la siguiente noticia: "Un comandante del ejército colombiano ordena a sus tropas duplicar la cantidad de criminales y rebeldes que matan, capturan u obligan a rendirse en batalla, y posiblemente acepte un aumento de las bajas civiles en el proceso, según consta en ordenes escritas y entrevistas con altos oficiales" (Casey, 18 de mayo de 2019). Desde la iniciación del conflicto armado colombiano, que duró más de 40 años, hasta hoy, las prácticas de algunos miembros del Ejército colombiano parecen no cambiar. Durante ese periodo, el enfrentamiento de grupos insurgentes con el Ejército Nacional y grupos paramilitares produjo un registro alto de víctimas y graves violaciones a los derechos humanos, entre los que se encuentran las ejecuciones extrajudiciales (Federación Internacional de Derechos Humanos, 2012). Sin embargo, fue después de la implementación de la política de seguridad democrática entre el 2002 y el 2010, cuando el número de ejecuciones extrajudiciales se incrementó a tal punto que ya no podía ser concebido como el resultado de eventos aislados, sino el de una política militar a nivel estatal. De acuerdo con la Oficina del Alto Comisionado para los Derechos Humanos de la ONU, entre el 2004 y el 2008 se habían reportado 3.000 víctimas mortales (Cárdenas \& Villa, 2013).

La Política de Seguridad Democrática, implementada a mediados del 2002 en el primer periodo de Álvaro Uribe Vélez, tenía como meta recuperar el control territorial debilitado por los intentos de diálogos de paz de administraciones pasadas, en los que se incluía el despeje del municipio del Caguán durante el gobierno de Andrés Pastrana (Cárdenas \& Villa, 2013). Bajo el marco de esa política de seguridad se otorgaron una serie de facultades a las fuerzas militares y un sistema de incentivos y recompensas para generar resultados visibles (Federación Internacional de Derechos Humanos, 2012). Este sistema carecía de mecanismos de control sobre las acciones de las fuerzas armadas bajo el supuesto de que no habría excesos, ignorando el papel que podrían tener los incentivos en el comportamiento de los mandos medios, quienes incitaban a los soldados a presentar bajas a cambio de ascensos y bonificaciones. Por supuesto, el resultado fue un incremento inusitado de víctimas mortales inocentes presentadas por militares como guerrilleros dados de baja en combate.

${ }^{1}$ La JEP es el componente de justicia del Sistema Integral de Verdad, Justicia, Reparación y no Repetición creado por el Acuerdo de Paz entre el Gobierno Nacional y las FARC-EP. Este componente dio a conocer, a través del Auto 033 de 2021, la primera fase de investigación del Caso 03: muertes ileǵtítimamente presentadas como bajas en combate por agentes del Estado. 
Este estudio intenta describir la naturaleza del problema e indagar por la responsabilidad de los altos mandos del Ejército colombiano en el aumento de falsos positivos durante la implementación de la política de seguridad democrática. Nuestra hipótesis es que el sistema de incentivos y recompensas, la fuerte estructura jerárquica del Ejército colombiano y la falta de castigos facilitaron la comisión de este delito.

Estudios sobre violencia unilateral y sexual de militares sobre civiles centran la responsabilidad en el soldado que actúa con el fin de conseguir beneficios adicionales, ocultándose de sus superiores (Humphreys \& Weinstein, 2006; Schneider et al., 2011; Butler et al., 2007, Mitchell, 2012). Por otro lado, Schneider (2011) y Schneider et al. (2011a) muestran que en algunos casos los incentivos establecidos por el comandante influyeron positivamente en el riesgo de abuso de los soldados contra la población civil.

Usando el análisis teórico que ofrece el modelo de principal-agente, el objetivo de este trabajo es estudiar la naturaleza de las ejecuciones extrajudiciales en Colombia, teniendo como hipótesis que estas acciones no fueron hechos aislados, sino que, por el contrario, los altos mandos del Ejército pudieron haber ordenado la comisión de esta clase de violencia unilateral contra la población civil a cambio de incentivos y castigos que alentaba a los soldados a realizar este tipo de operaciones.

Nuestros hallazgos muestran que el castigo impuesto por el principal es un determinante clave en la decisión de cometer una ejecución extrajudicial. Este resultado confirma la hipótesis de que los altos mandos del Ejército colombiano, además de ordenar los falsos positivos, también influyeron, mediante mecanismos de castigo, en la decisión de los agentes de cometer este tipo de homicidios. El resto del trabajo continua así: la siguiente sección contiene una reseña de literatura reciente sobre violencia unilateral en tiempos de guerra y sus principales determinantes; en las secciones subsiguientes se describen el diseño teórico y los resultados; en la sección final se presentan las conclusiones.

\section{Revisión de literatura}

Un número creciente de estudios muestra que la forma en que operan las unidades militares y su estructura jerárquica influye en el uso de la violencia contra civiles. Algunos estudios explican que la violencia unilateral se debe a la ausencia de mecanismos punitivos dentro de las unidades militares y gubernamentales (Humphreys \& Weinstein, 2006; Schneider et al., 2011b). Por ejemplo, Butler et al. (2007) usan un enfoque de principal-agente para analizar datos transnacionales sobre la incidencia de abusos sexuales en el año 2003 cometidos por las fuerzas militares contra la población civil, mediante el cual mostraron que la violencia sexual fue el resultado de las decisio- 
nes de los propios agentes, quienes, actuando a espaldas de sus jefes, cometieron actos sexuales violentos contra la población como una forma de recompensa por vía propia. La violación de mujeres jóvenes se convirtió en un beneficio adicional. Esta clase de comportamientos tiende a ser más frecuente en países inmersos en conflictos civiles, en los que es más fácil para los agentes ocultar sus acciones y donde el reclutamiento puede generar problemas de selección adversa.

En estos enfoques la responsabilidad recae en los combatientes y no en sus superiores. Sin embargo, ellos son culpables al no implementar castigos contra desviaciones de la conducta esperada de sus agentes. Así, la violencia contra civiles no se limita solo a fallas en la estructura de la organización o a la ausencia de mecanismos de control, sino a la presencia de incentivos que permiten que los soldados tiendan a valorar más el asesinato de civiles frente a otro tipo de acción (Mitchell, 2004; Schneider, 2011). Los superiores que también se ven beneficiados manipulan las necesidades de los soldados de asegurar ingresos adicionales, para así volverlos asesinos y mejorar los resultados. Un ejemplo es el caso de la violencia sexual generalizada en la República Democrática del Congo; durante muchos años este país estuvo sumido en una devastadora guerra civil caracterizada por violaciones y agresiones sexuales contra las mujeres (Schneider et al., 2011b).

Para DeMeritt (2015), el asesinato de civiles no es causado solo por agentes descontrolados ni tampoco es consecuencia únicamente de los incentivos ofrecidos por los líderes, la violencia unilateral también puede provenir de una política de Estado que envía una orden y de un agente que la implementa. En muchos casos resulta costoso desobedecer por el castigo que podría recibirse. A nivel de la violencia urbana, Ahnen (2007) examina los homicidios policiales en 19 estados brasileños entre 1994 y 2001 y muestra que las medidas de seguridad pública que tienen énfasis en el uso de la fuerza pueden causar violaciones de derechos humanos por parte de la Policía. Feaver (1999) muestra que los incentivos prometidos para que los soldados ejecuten su labor corrompe la unidad militar y la lealtad a las autoridades contra la población civil. A nivel del país, las ejecuciones extrajudiciales son un claro ejemplo de violencia unilateral; su incremento se produjo durante la implementación de la Política de Seguridad Democrática. Cárdenas y Villa (2013), usando un modelo de principal-agente, muestran que tal incremento estaría relacionado con los incentivos que el Gobierno ofreció a las fuerzas militares, y proponen, para evitar ese tipo de conductas, una política de seguridad alternativa que incluya mecanismos de control por parte del Gobierno. 


\section{Ejecuciones extrajudiciales en Colombia}

El fenómeno de los falsos positivos no es nuevo, por el contrario, lleva décadas golpeando al país. El Centro de Investigación y Educación Popular (CINEP) (2010) identifica 1.200 casos de falsos positivos ocurridos en Colombia entre 1988 y 2011. Sin embargo, durante el gobierno de Álvaro Uribe Vélez, el número de casos creció, dejando un saldo de 500 víctimas por falsos positivos en su primer periodo presidencial y de casi 700 para el segundo (CINEP, 2011; Noticias RCN, 2014), en 30 departamentos. Uno de los hechos más emblemáticos fue el caso de Soacha y Ciudad Bolívar en Bogotá, donde el reporte de las familias por la desaparición de 19 jóvenes coincidió con el informe de las fuerzas militares de que habían sido abatidos en combate guerrilleros en Ocaña, Norte de Santander (CINEP, 2011). En el 2021, la JEP declara que hubo por lo menos 6.402 personas muertas ilegítimamente y presentadas como bajas en combate en Colombia en el periodo 2002 al 2008, principalmente en Antioquia, la Costa Caribe, Norte de Santander, Huila, Casanare y Meta.

Como afirma Londoño Berrío (2018), aunque existen registros de ejecuciones extrajudiciales en el país desde tiempo atrás, fue en el periodo 2002-2010 en el que se presentó el mayor número de falsos positivos de forma inusitada y sistemática. El aumento de las ejecuciones extrajudiciales durante el periodo de la seguridad democrática fue el resultado de la presión ejercida por los altos y medios mandos para mostrar resultados, expresada en la política de conteo de cuerpos incorporada por el Gobierno para medir la efectividad de las Fuerzas Armadas Colombianas. Las recompensas monetarias para quienes mostraran las bajas constaban de hasta 10 salarios mínimos legales vigentes por cada guerrillero abatido en combate, además de beneficios adquiridos, como licencias y cursos en el exterior (CINEP, 2011). En ese sistema, las recompensas para los reclutadores estaban entre 200.000 y 1.000 .000 de pesos por cada joven reclutado para ser asesinado, mientras que los altos mandos militares y los comandantes de unidad recibían entre 2 y 5 millones de pesos por cada "baja", además de descansos remunerados (Rojas-Bolaños \& Benavidez-Silva, 2017).

La impunidad jugó un papel decisivo. En el 2010 la tasa de impunidad para los asesinatos atribuidos a las fuerzas armadas colombianas era del $98,5 \%$, lo que hacía más atractivo el cometer un falso positivo (Alston, 2010; Lindsay-Poland, 2014). Además, existía un código de silencio dentro de las unidades militares para ocultar este tipo de hechos. Ante el castigo por delación, los que eran atrapados cometiendo este tipo de delitos preferían asumir públicamente su responsabilidad antes que denunciar a un superior. Esta clase de conductas, junto con la inoperancia de la Justicia Penal Militar, sirvieron de blindaje para todas aquellas unidades militares que cometieron violaciones de derechos humanos (Rojas-Bolaños \& Benavides-Silva, 2017). Esto se sumó a la negativa del Gobierno de reconocer el fenómeno delictivo, afirmando que se trataba de casos ais- 
lados (Gómez, 2011). En una intervención en la Asamblea de ANIF, el expresidente de la república, Álvaro Uribe Vélez, afirmó, al referirse al caso de jóvenes desaparecidos en Soacha, que "no fueron a recoger café, iban con motivos delincuenciales" (El Espectador, 7 de octubre de 2008).

El modus operandi para un falso positivo es documentado por Rojas-Bolaños y BenavidesSilva (2017). Antes de cometer el falso positivo se realizaban operaciones llamadas psicosociales, en las que se difundía información falsa para fortalecer la confianza en la efectividad de las fuerzas armadas. A nivel de las unidades operativas, se organizaban reuniones en que autorizaban los combates militares a través de documentos oficiales, y se establecía qué recursos eran necesarios para cometer un falso positivo, el lugar del operativo y el reclutamiento de las potenciales víctimas. También se determinaba la forma en que sería asesinada la víctima, quién la vestiría y quiénes serían los encargados de ubicarla en la escena del crimen. Se delegaban tareas relacionadas con los elementos de guerra, la incautación de propaganda subversiva y la realización de disparos que alertarían a los residentes de la región sobre el combate que supuestamente estaba ocurriendo. Después, se llevaría a cabo el acordonamiento del lugar de los hechos, la entrega de elementos incautados y el levantamiento de las víctimas, además, debía realizarse una presentación de los hechos ante los medios. Finalmente, se hacía la entrega de recompensas, licencias, vacaciones pagas, entre otras (Rojas-Bolaños y Benavides-Silva, 2017).

\section{¿Qué tan responsables eran los altos mandos?}

Para establecer el papel que tuvieron los altos mandos de las fuerzas militares y su estructura jerárquica en el aumento de ejecuciones extrajudiciales durante la política de seguridad democrática, se deben considerar los siguientes elementos:

\section{Negligencia y falta de control como norma}

En varios de los casos de falsos positivos hubo documentos que contenían instrucciones de cómo se debían llevar a cabo las operaciones -conocidas como "misiones tácticas" y órdenes de operaciones "fragmentarias"-, que justificaban legalmente los desplazamientos de los soldados a las zonas donde se cometían los crímenes (Human Rights Watch, 2015) y cuyas bajas en supuestos combates eran notificadas a los superiores. Para Human Rights Watch, las circunstancias en las que estas se presentaban debieron advertir a los comandantes sobre presuntos falsos positivos.

Para el caso de Soacha, la investigación realizada por una comisión transitoria del Ministerio de Defensa mostró que las unidades militares que operaban en Santander, junto con otras como la XIV Brigada de Antioquia, cometieron faltas graves de control por parte de los más altos ran- 
gos, relacionadas con la informalidad excesiva en la presentación de los resultados, la omisión de requisitos de planeación y autorización para las operaciones militares realizadas en esa zona (Gómez, 2011).

\section{El body counting y la presión por bajas}

Aparte del conocimiento de los actos delictivos cometidos dentro de la unidad militar, estaba la presión por bajas ejercida por los comandantes de brigada, fundamentada en las políticas de un Gobierno que exigía resultados. Fabre (2015) llama a este fenómeno "síndrome de conteo de cuerpos".

Parte de las presiones ejercidas en las unidades militares estaba dada por las cuotas de bajas impuestas en las diferentes brigadas y por las competencias con las que determinaban cuál de estas lograba la mayor cantidad de bajas (Human Rights Watch, 2015). Un ejemplo de ello es el testimonio de Robinson González del Río sobre el general Mario Montoya, quien es conocido por su famosa insistencia en querer ver "litros de sangre"; este general instauró un ranking con el que las unidades militares eran medidas a través de un top de bajas que las priorizaba sobre las capturas (Arenas, 2015).

El general Montoya impuso una serie de políticas con las que presionaba a los militares bajo su mando para que presentaran resultados operacionales. En la orden del día N.73 del miércoles 5 de abril de 2006, se establecieron las tareas cotidianas del Batallón de Ingenieros Pedro Nel Ospina, localizado en Bello, Antioquia, recordando que estas eran "órdenes de carácter permanente" y debían ser tenidas en cuenta no solo ahí, sino en todas las unidades militares del país (Verdad Abierta, 13 de septiembre de 2017). Frases como, por ejemplo, "Los comandantes se evalúan por sus resultados" y "las bajas no son lo más importante, son lo único", impusieron una fuerte presión a las tropas. Otro testimonio es el de Luis Fernando Borja, comandante de la Fuerza de Tarea Conjunta de Sucre, quien asegura que aquellos comandantes que no cumplían la cuota de bajas eran "reprendidos, trasladados o retirados" de las unidades militares (El Espectador, 16 de abril de 2016).

Human Rights Watch (2015) muestra evidencia de cómo los superiores amenazaban con sancionar aquellos soldados que no produjeran bajas en combate. Estas represalias también servían de advertencias, para otros posibles testigos, sobre no declarar o silenciar también a familiares de las víctimas. Por otro lado, los militares que se han atrevido a denunciar ejecuciones extrajudiciales perdieron la vida en enfrentamientos ficticios contra grupos terroristas (Rojas-Bolaños \& Benavides-Silva, 2017). Además, existen casos como el del sargento Carlos Eduardo Mora, quien al negarse a participar en los crímenes e intentar denunciarlos fue víctima de amenazas por 
parte de sus superiores (Human Rights Watch, 2015). Todos estos aspectos dificultaban cualquier testimonio por parte de soldados que no estuvieran de acuerdo con esta clase de abusos, quienes, por su propia seguridad, se veían obligados a reubicarse (Alston, 2010).

\section{Obediencia ciega}

Además de la presión por bajas, la obediencia ciega por parte de los soldados jugó un papel importante en el aumento de falsos positivos. Los incentivos fortalecieron dicha lealtad, por lo que las bajas se presentaban a cambio de no ser relevado, de ser llamado a curso, de ser tenido en cuenta para la entrega de estímulos y premios. Rojas-Bolaños y Benavides-Silva (2017) muestran testimonios que corroboran este hecho; por ejemplo, el de un oficial involucrado en casos de falsos positivos, quien afirmaba que "de no hacerlo, simplemente sería un militar insignificante, sin medallas de orden público, sin trayectoria en la guerra, sin nada que mostrar para cuando me llamaran a evaluarme para general". Además, agrega: "nos ordenaron muertos y muertos les dimos, un general nos pedía litros de sangre y se los dimos, una orden es una orden y se va al ejército es para obedecer, para cumplir órdenes, no para refutarlas". Para el 2017 existían más de 5.000 militares comprometidos en casos relacionados con ejecuciones extrajudiciales (Rojas-Bolaños \& Benavides-Silva, 2017).

\section{Impunidad}

En primer lugar, el incentivo económico y las diferentes bonificaciones creaban lealtad de los militares hacia la institución, lo que terminaría fomentando la impunidad debido a que no habría testimonios que incriminaran a la institución militar ni a los altos mandos. Por otro lado, aquellos que quisieran denunciar debían asumir las consecuencias de las amenazas, muertes y destituciones. La persecución contra los fiscales encargados de ejecuciones extrajudiciales también ayudó a que este tipo de acciones no salieran a la luz pública, así como la actitud adoptada por los medios de comunicación. Según Rodríguez (2012), la forma sesgada en que los medios cubrían estos acontecimientos afectaba la reparación de las víctimas de falsos positivos; este autor analizó el cubrimiento del caso de ejecuciones extrajudiciales en Soacha realizado por el periódico El Tiempo, desde septiembre de 2008 hasta agosto de 2009, y mostró que, en su mayoría, la voz principal era la de algún funcionario del Gobierno o representante de las fuerzas armadas y que tan solo en 7 de los 90 artículos la opinión principal pertenecía a una víctima.

Por último, la justicia penal militar sirvió de blindaje para aquellos militares que se vieron envueltos en acciones ilegales (Rojas-Bolaños \& Benavides-Silva, 2017). Según Semana (23 de abril de 2015), 27 altos mandos involucrados en ejecuciones extrajudiciales no pagaron condena, tan solo fueron separados de las fuerzas armadas, y solo 6 coroneles han sido condenados. Fi- 
nalmente, ningún general fue procesado y mucho menos condenado. Hasta el 2018, aproximadamente 1.200 oficiales y suboficiales están cumpliendo condena por falsos positivos; sin embargo, los generales no han sido condenados por dichos delitos (Vivanco, 19 de marzo de 2018). Las dilaciones y prórrogas en los procesos judiciales han llevado a que muchos de los implicados en ejecuciones extrajudiciales estén libres por vencimiento de términos, como el caso de los 17 implicados en el caso de Soacha, que para el 2015 aún seguían en libertad (Caracol Radio, 21 de febrero de 2013), o que algunos estén asumiendo cargos de mando en el Gobierno a pesar de estar siendo investigados por la fiscalía. De acuerdo con Human Rights Watch (2019), el gobierno del presidente Duque ha nombrado a nueve generales contra los que existía evidencia que los involucraba en ejecuciones extrajudiciales: "Al nombrar a estos generales, el gobierno transmite a las tropas el preocupante mensaje de que cometer abusos puede no ser un obstáculo para avanzar en la carrera militar" (Human Rights Watch, 2019).

Ahora bien, considerar las ejecuciones extrajudiciales como consecuencia de acciones individuales adjudicadas a unos cuantos miembros de las fuerzas militares proporciona una explicación limitada al fenómeno de los falsos positivos. Como lo muestra Alston (2010), tantos casos similares hacían imposible pensar que fueran hechos aislados. A lo largo del país, esta clase de crímenes fueron cometidos exhibiendo un patrón similar cuya organización involucraba a más de una entidad dentro del Ejército; el modus operandi relacionado con los falsos positivos requería de una elevada planeación y organización (Human Rights Watch, 2015).

\section{Metodologia}

El método descrito aquí propone un enfoque teórico del modelo principal-agente que sugiere la existencia de un principal que contrata a un agente para realizar unas tareas bajo un contexto de información imperfecta, en el que el principal tiene que ofrecer al agente incentivos para que trabaje más y se alcance su mayor beneficio. Este modelo permite explicar teóricamente el comportamiento asumido por miembros de las fuerzas armadas que ejercieron una violencia unilateral expresada en ejecuciones extrajudiciales de población civil. En este enfoque son importantes los incentivos generados por el Gobierno, junto con los generados por los comandantes al interior de la unidad militar (como principal) para que los soldados (como agentes) cometieran este tipo de asesinatos extrajudiciales. También se pretende capturar los rasgos principales del fenómeno de los falsos positivos teniendo en cuenta la responsabilidad de los comandantes de las unidades de contraguerrilla. 
Se tomará como aproximación metodológica el modelo de DeMeritt (2015) y el de Cárdenas y Villa (2013). El primero incorpora aspectos que explican la responsabilidad de los altos mandos en el aumento de falsos positivos, modelando los castigos en función del body counting, tanto los impuestos por el principal, por el Gobierno y la comunidad internacional, como los beneficios esperados. Así mismo, tiene en cuenta la preocupación del principal por su legitimidad y por mantener intacta la jerarquía. Sin embargo, el modelo de DeMeritt no considera las bonificaciones y otros beneficios que recibían los agentes por cometer esta clase de crímenes, aspecto característico de los falsos positivos en Colombia.

En ese sentido, el modelo de Cárdenas y Villa (2013), también bajo un enfoque de principal agente, muestra la relación que existe entre esa política y el incremento en ejecuciones extrajudiciales. El principal es el Gobierno, cuyo objetivo es alcanzar un nivel máximo de bienestar para la sociedad. Dicho bienestar está determinado por el debilitamiento de los grupos armados al margen de la ley que se expresa por las bajas en combate. Sin embargo, el bienestar de la sociedad va a decrecer cada vez que aumente el número esperado de falsos positivos y el costo de supervisión de la unidad militar. Todo esto es representado en una función de bienestar $B$ reducida,

$$
B=U(g)+D(f)+S-C
$$

En la ecuación, $g$ es el número de bajas esperadas en combate de los grupos armados ilegales y $f$ el número de falsos positivos; $S$ es un parámetro exogéno que refleja la sanción a los militares que tengan conductas ilegales; por último, $C$ es el costo de supervisión del agente. Además, existe una probabilidad $q$ de que los agentes sean detectados cometiendo falsos positivos. De esta forma, el mecanismo propuesto por los autores para la política de seguridad democrática está dado por $M^{P S D}=(R, \bar{q}, \bar{S})$, donde se toman como dados tanto $S$ como $q$, y el Gobierno solo usa $R$, que son los recursos necesarios para la implementación de la política que debilite a los grupos armados ilegales.

Además, se modelan factores característicos de esta política, como la tributación necesaria para financiarla y el personal para llevar a cabo dicha tributación. Además, se formaliza el comportamiento tanto del Gobierno como de la unidad militar. Por último, los resultados se contrastan con los de un modelo en el que se refuerza el sistema de monitoreo, de esta forma, el Gobierno no solo usaría $R$ para lograr su objetivo, sino que también tendría en cuenta $q$, de tal forma que el mecanismo sería $M^{P S D}=(R, q, \bar{S})$. Cárdenas y Villa (2013) concluyen que esta última propuesta de política reduce el problema de las ejecuciones extrajudiciales y que, aunque el nuevo nivel de auditoría podría resultar costoso para el Gobierno, ayuda a incrementar el bienestar social, ya que existe una mayor probabilidad de detección de falsos positivos. 
Partiendo del planteamiento de DeMeritt (2015) se modela el fenómeno de los falsos positivos con base en el trabajo de Cárdenas y Villa (2013). En este trabajo, los falsos positivos no serán tratados como hechos aislados, sino como el resultado de la presión por bajas por parte de los altos mandos del Ejército. Debido a la fuerte estructura jerárquica de las fuerzas armadas colombianas, el militar no solo estaba impulsado por su beneficio personal, sino también por la presión del comandante y por el probable castigo por desobediencia, que actuaba como un incentivo más para llevar a cabo el asesinato de civiles ${ }^{2}$. Además, aspectos como el castigo judicial o internacional por dicho crimen pueden afectar la elección del militar entre obedecer o desobedecer la orden.

\section{El juego}

Se parte aquí del supuesto de que los altos mandos del Ejército colombiano no desconocían que este tipo de crímenes estaban ocurriendo. Es conveniente subrayar que entre 2002 y 2008 más de 150 batallones y unidades tácticas pertenecientes a 41 brigadas, subscritas a 7 divisiones existentes, habían cometido falsos positivos (Human Rights Watch, 2015). Por tanto, el juego planteado es entre los altos mandos representados por el comandante del Ejército nacional y otros comandantes subordinados, como los de brigada y unidades tácticas, y los soldados o mandos no superiores como autores materiales de los falsos positivos. Esta interacción ocurría en un contexto en el que las unidades ejercían presión por bajas, motivadas por el incentivo económico procedente de la política de seguridad democrática y por la imposición de un castigo o tomando represalias contra aquellos que no cumplieran órdenes. En este juego se asume que la probabilidad de ser encontrado culpable y judicializado es mayor para un soldado que para un alto mando del Ejército ${ }^{3}$.

\section{Movimientos}

Se supone que, al igual que en el modelo de DeMeritt (2015), el comandante elegía un número de bajas ideal para obtener beneficios. Ese número estaría determinado por las metas impuestas en las diferentes brigadas, buscando situarse en los primeros puestos de las estadísticas ${ }^{4}$. El juego estaría diseñado de la siguiente manera:

${ }^{2}$ En el Apéndice D se muestra la intuición gráfica del mecanismo relacionado con las ejecuciones extrajudiciales en Colombia.

${ }^{3}$ Human Right Watch (2015) muestra que el número de soldados condenados es mayor al número de altos mandos, de igual forma, el castigo para un comandante 0 un coronel resulta ser mucho menos severo.

${ }^{4}$ Estadísticas que respondían a los rankings implementados por altos mandos, como el coronel William Hernán Peña Forero, según el testimonio del comandante Luis Fernando Borja (El Espectador, 16 de abril de 2016). 


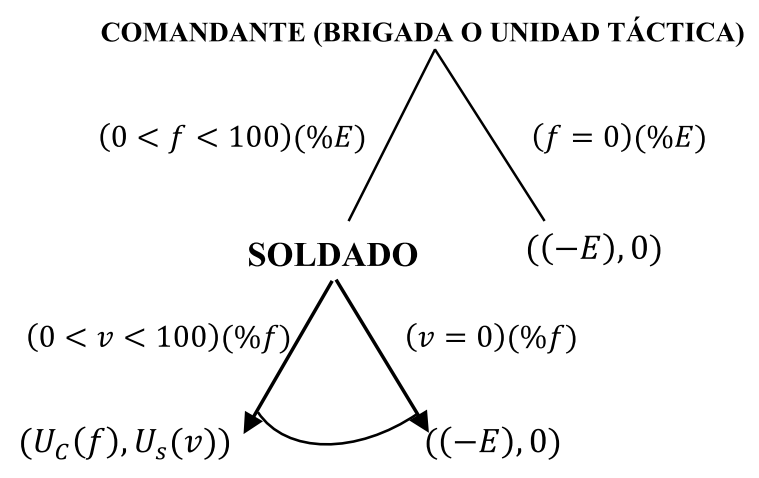

\section{Movimientos del comandante (principal)}

Se va a suponer que existe un top 10 de las unidades con más bajas y una presión por resultados impuesta desde el comandante del Ejército nacional hacia los comandantes de brigada y unidades tácticas ${ }^{5}$. Siguiendo la metodología de DeMeritt (2015), el comandante de brigada o unidad táctica evaluará su situación en cuanto al número de bajas que necesita para adquirir diferentes clases de premios, bonificaciones, ascensos, entre otros. Elegirá un porcentaje ideal de falsos positivos dentro de su conteo de bajas: $0<\underline{\mathrm{E}}<100$; por tanto, $\mathrm{E}=$ (Cuotas de bajas - Positivos llevados a cabo). De esta forma, cuando la cuota de bajas en combate es alcanzada por medio de positivos, no será necesario cometer ejecuciones extrajudiciales; sin embargo, cuando no se logran realizar positivos, toda la cuota de bajas en combate será alcanzada asesinando civiles. Después de elegir un porcentaje ideal de falsos positivos, los comandantes de brigada y unidad táctica ordenarán a los soldados cometer un porcentaje del número ideal de ejecuciones extrajudiciales: $0<\mathrm{f}<100$. Vamos a suponer que este será el número de falsos positivos que los soldados deberán llevar a cabo por orden de sus superiores.

\section{Movimientos del soldado (agente)}

El soldado cometerá, por orden del comandante, un porcentaje del número de falsos positivos que le son ordenados: $0<\mathrm{v}<100$. A diferencia de DeMeritt (2015), en este modelo, el principal no es el Gobierno, sino un alto mando del Ejército que está motivado por beneficios monetarios, más que por objetivos militares o políticos. Esto tiene implicaciones importantes, ya que, aparte del castigo internacional propuesto por DeMeritt (2015), también va a existir el riesgo de ser capturado y posteriormente judicializado. Tanto el comandante como el soldado tienen que incluir este costo probable en su función de utilidad. Además, el modelo de DeMeritt (2015) no incluye

${ }^{5}$ Un ejemplo de ello es el general Mario Montoya, quien fue comandante del Ejército nacional entre febrero de 2006 y noviembre de 2008, y era reconocido por establecer cuotas de bajas en combate y por ejercer, además, presión por bajas sobre comandantes subordinados (Human Rights Watch, 2015). 
ninguna remuneración económica, pues el principal es el Gobierno y los agentes deben cumplir con las órdenes que les son impuestas. Sin embargo, para el caso de los falsos positivos, además del cumplimiento del deber, existe un incentivo económico adicional por bajas que también debe ser incluido en la función de utilidad, del principal y del agente.

\section{Supuestos}

Entre los supuestos se incluyen algunos del modelo de DeMeritt (2015) y otros que tienen que ver con particularidades del fenómeno estudiado.

- S1: Los soldados valoran su vida por encima de la de los demás.

- S2: Las ejecuciones extrajudiciales son usadas para beneficios individuales.

- S3: Los altos mandos castigan la desobediencia con probabilidad $p=1$. Sin embargo, no en todos los casos los altos mandos observan la desobediencia de manera perfecta, por lo que el soldado podría no cumplir la orden siempre que logre evitar ser descubierto por su superior. Por tanto, existirá una proporción esperada de la desobediencia observada por el alto mando, $a \in[0,1]$.

- S4: la probabilidad de captura y condena por un falso positivo por parte de las autoridades nacionales es mayor para el agente que para el principal ${ }^{6}$.

Tabla 1

Nombre de variables usadas en el modelo

\begin{tabular}{cl} 
Variable & Nombre de variable \\
\hline$E>0$ & Número ideal de bajas de civiles que permitirá a la brigada llegar a los primeros puestos de las estadísticas \\
\hline$a \epsilon[0,1]$ & Proporción esperada de la desobediencia observada por el líder de la unidad militar \\
\hline$i \epsilon[0,1]$ & Probabilidad de castigo internacional por asesinar civiles \\
\hline$q_{c} \epsilon[0,1]$ & Probabilidad de que el alto mando sea condenado por la justicia colombiana por cometer falso positivo \\
\hline$q_{m} \epsilon[0,1]$ & Probabilidad de que el soldado sea condenado por la justicia colombiana por cometer falso positivo \\
\hline$c>0$ & Costo en el que incurre el líder de la unidad militar al castigar la desobediencia \\
\hline$d \epsilon[0,1]$ & Preocupación de los altos mandos por su propia legitimidad \\
\hline$p>0$ & Violencia esperada del castigo por desobedecer una orden \\
\hline $0<\mathrm{V}<100$ & Porcentaje de falsos positivos que son cometidos por el soldado \\
\hline $0<\mathrm{f}<100$ & Porcentaje de falsos positivos que se le ordenan cometer al soldado \\
\hline
\end{tabular}

${ }^{6}$ Según José Miguel Vivanco, director de la ONG Human Right Watch, tan solo 4 generales han sido investigados, e incluso algunos han sido ascendidos (Vivanco, 19 de marzo de 2018). 


\section{Función de utilidad del comandante}

Para el comandante del Ejército Nacional, los falsos positivos representan diferentes beneficios que van desde lo económico (ascensos y capacitaciones) hasta la percepción de que se estaba cumpliendo con las expectativas del Gobierno. Ellos deben garantizar la obediencia y el cumplimiento de cada una de las órdenes, porque de esta manera mantienen su legitimidad y ratifican su mando dentro de la unidad militar.

Basados en DeMeritt (2015), estos aspectos serán representados en una función de utilidad o beneficio del comandante de brigada por ordenar cometer ejecuciones extrajudiciales. El hecho de que las funciones sean negativas muestra que a medida que las ejecuciones extrajudiciales cometidas se acerquen al número ideal, la utilidad del alto mando será mayor. De igual forma, a medida que el número de falsos positivos cometidos se acerque al número ordenado por el alto mando, la utilidad del soldado será mayor.

$$
\begin{aligned}
& 1 \quad 2 \quad 3 \quad-4 \quad 5 \\
& U c(f)=-(E-v)^{2}-i v^{2}-d(f-v)^{2}-a c(f-v)^{2}-q_{c}(v)^{2} .
\end{aligned}
$$

El primer componente de la función (1) son los beneficios percibidos por el comandante cuando alcanza la meta requerida para estar en los primeros puestos del ranking o de las estadísticas: $E$. El segundo componente representa los costos referentes al castigo; a medida que el número de bajas aumenta, la probabilidad de ser atrapados y castigados por instituciones internacionales aumenta; por tanto, a medida que se incrementa la probabilidad de ser detectado por la comunidad internacional, cometer falsos positivos se vuelve más costoso.

Por otro lado, la desobediencia es costosa, no porque no permita alcanzar el objetivo propuesto, sino porque disminuye la legitimidad del comandante y su monopolio del poder dentro de la brigada. El peso de este costo está dado por $d \epsilon[0,1]$ y variará dependiendo del comandante. Para un comandante que considere que la desobediencia no afecta su legitimidad, su monopolio de poder o su situación legal, este componente tomará el valor de 0 . Si, por el contrario, el comandante considera que la desobediencia puede conllevarle problemas legales o afectar su legitimidad, el valor de este componente será de 1 .

El cuarto componente muestra los costos de castigar a un soldado después de observar $v$, es decir, después de saber si cumplió o desobedeció la orden. Por lo tanto, cuando $a=0$, el comandante no va a incurrir en costos de castigo. Por el contrario, cuando $a=1$, el comandante estará en la facultad de hacer un seguimiento a sus tropas para observar la desobediencia e imponer un castigo. El costo $c$ de llevar a cabo dicho castigo es $c>0$. De esta forma, cuando $c=0$, no habrá castigo, y aumentará a medida que aumenta la diferencia entre falsos positivos cometidos y ordenados. 
Como no se tiene evidencia de cuáles acciones cometidas por las fuerzas armadas colombianas son ilegales, o cuáles fueron apoyadas por el Gobierno, entonces, además del castigo internacional es necesario incluir el castigo por parte de autoridades nacionales. El quinto componente representa la probabilidad de recibir dicho castigo, a medida que la probabilidad de ser observados por las autoridades aumenta, cometer falsos positivos se vuelve mucho más costoso.

\section{Función de utilidad del soldado}

Los soldados valorarán su propia integridad y sus propios intereses por encima de la vida de los civiles. Por tanto, la utilidad del soldado va a estar determinada, en primer lugar, por el incentivo económico, pero, en segundo lugar, por el interés de proteger su propia vida y su libertad.

Por ejemplo, no obedecer la orden o estar en desacuerdo representan un riesgo para los altos mandos, ya que, en primer lugar, no solo van a perder su legitimidad, sino que aumenta la probabilidad de ser delatados por estos soldados desobedientes. Por tanto, el comandante buscará castigar la desobediencia. Sin embargo, al igual que en el modelo de DeMeritt (2015), el costo de desobedecer depende de la capacidad del comandante para detectarla. Además, este costo responde en gran medida a la percepción del castigo que tenga el soldado; si el soldado cree que será asesinado o tendrá castigos que atenten contra su integridad física, será más costoso para él desobedecer la orden de cometer un falso positivo. Por tanto, su función de utilidad estará representada por:

$$
\begin{aligned}
& 1 \quad 2 \quad 3 \quad-4 \\
& U s(v)=-a p(f-v)^{2}-i v^{2}-q_{m}(v)^{2}-(f-v)^{2}
\end{aligned}
$$

El primer componente describe el costo de desobedecer. A medida que la desviación $(f-v)^{2}$ aumenta, el costo será mayor. El segundo componente tiene que ver con la probabilidad de ser atrapado y castigado por la comunidad internacional. El tercero capta la probabilidad de ser atrapado y castigado por autoridades nacionales, siendo mayor para el agente que para el principal, quien, en primer lugar, goza de mayor poder y contactos, y en segundo lugar, a pesar de ordenar y presionar para cometer falsos positivos, no es autor material del delito y puede llegar a pasar desapercibido. 
El último componente muestra las bonificaciones obtenidas cuando se alcanza la meta de bajas ordenada por el alto mando ${ }^{7}$, que será mayor a medida que aumente el número de bajas. Debido a que el Gobierno solo observa el número de bajas y no logra diferenciar entre bajas en combate y de civiles, el incentivo será entregado independientemente de las circunstancias de la baja.

\section{Equilibrio}

El equilibrio se obtiene maximizando la función de utilidad del soldado con respecto al número de bajas que llevará a cabo (v). De esta forma, obtenemos la mejor respuesta del soldado a la orden impuesta por su superior. La derivación formal de este equilibrio se puede encontrar en el Apéndice A. En este punto, el soldado escoge el número de bajas que maximice su utilidad en función de los parámetros exógenos del juego $\left(a, q_{m}, q_{\mathcal{c}^{\prime}} p, i \mathrm{y} w\right)$ obteniendo:

$$
v^{*}=\frac{f(a p+1)}{\left(q_{m}+a p+i+1\right)}
$$

\section{Análisis de la ecuación (3)}

Cuando existe una probabilidad positiva de ser observado al desobedecer la orden y de ser castigado, la elección del soldado dependerá del número de civiles que se le haya ordenado asesinar. De esta forma, cuando a los soldados no se les ordena cometer ejecuciones extrajudiciales, es $\operatorname{decir} f=0$, el número de bajas será 0 . Por otro lado, a medida que $f$ aumenta, el soldado cometerá más asesinatos de civiles y el número de ejecuciones extrajudiciales aumentará. La elección óptima del soldado está influenciada exógenamente por factores como la posibilidad de ser observado por sus superiores o que su desobediencia sea castigada. Por tanto, si $a=0$ y $p=0$, los superiores no pueden observar las acciones de los soldados y estos no podrán ser castigado por desobedecer. En este caso, el número de bajas dependerá del número de ejecuciones extrajudiciales que sean ordenadas y de qué tan alto sea el castigo. Así, a medida que $a$ y $p$ aumenten, desobedecer la orden será menos atractivo y las ejecuciones extrajudiciales se incrementarán. Este modelo se diferencia del de DeMeritt (2015) en que en ausencia de castigo por desobediencia, aunque las bajas disminuyen, estas no desaparecen, debido a la presencia del incentivo económico para el soldado, que afecta sus decisiones.

${ }^{7}$ Entre estas se encuentran las otorgadas por el decreto 029 de 2005 del Ministerio de Defensa, que fijaba criterios para el pago de recompensas por captura 0 abatimiento en combate de cabecillas de grupos armados al margen de la ley, información sobre narcotráfico, material de guerra y pago de información que pudiera servir para labores de inteligencia y para el planeamiento de operaciones (Martín-Córdoba, 2015). 
Por otro lado, la impunidad es importante. Cuando los soldados estiman que la probabilidad de ser penalizado internacionalmente o por las autoridades nacionales es $0\left(q_{m}=0, i=0\right)$, el soldado cometerá tantas ejecuciones extrajudiciales como le sean ordenadas $\left(v^{*}=f\right)$. A medida que aumente la probabilidad esperada de ser castigado penalmente, el número de bajas de civiles disminuirá.

Ahora bien, consideremos la decisión que enfrenta el alto mando del Ejército. Siguiendo a DeMeritt (2015) diremos que $v=z f$, donde $z=\frac{a p+1}{q_{m}+a p+i+1}$, con el objetivo de facilitar la derivación. Dada la elección óptima del soldado, la función de utilidad del comandante será:

$$
U c(f)=-(E-z f)^{2}-i(z f)^{2}-d(f-z f)^{2}-a c(f-z f)^{2}-q_{c}(z f)^{2}
$$

Y su elección óptima estará determinada por:

$$
f^{*}=\frac{E z}{z^{2}\left(1+q_{c}+i\right)+(d+a c)(1-z)^{2}}
$$

La decisión que enfrenta el comandante, junto con la elección óptima del soldado, conforman la única estrategia pura del juego; por tanto, el equilibro perfecto en subjuegos es:

$$
\left\{v^{*}=\frac{f(a p+1)}{\left(q_{m}+a p+i+1\right)}, f^{*}=\frac{E z}{z^{2}\left(1+q_{c}+i\right)+(d+a c)(1-z)^{2}}\right\} .
$$

La elección óptima del soldado puede llevar a tres situaciones. En primer lugar, existe la posibilidad de que no se realicen ejecuciones extrajudiciales; en segundo lugar, existe una situación en la que el soldado realizará exactamente el número de bajas que le sea ordenado y; por último, que estos cumplan la orden, pero en distintos grados. Posteriormente, se va a analizar cada una de ellas para discutir las diferentes implicaciones de este modelo.

\section{Estática comparativa}

El modelo nos muestra que la única forma de que no haya ejecuciones extrajudiciales es que no se dé la orden para cometerlas. Sin embargo, es necesario considerar el efecto que tienen todos los demás factores exógenos en el número de falsos positivos que cometerá el soldado. Se analizará cuál es la lógica detrás de las ejecuciones extrajudiciales cuando están en función de la orden del alto mando y de un conjunto de factores exógenos. Siguiendo la metodología de DeMeritt (2015), se obtendrán segundas derivadas parciales de la utilidad del alto mando con respecto a cada parámetro exógeno para medir su efecto en $f^{*}$. Con $\frac{\partial^{2} U s(v)}{\partial f}$ estrictamente positiva; entre más falsos positivos ordenen los altos mandos, más de estos crímenes se cometerán. 
Factores como el costo de castigar la desobediencia y la preocupación del alto mando por mantener su legitimidad están relacionados negativamente con el número de falsos positivos ordenados. A medida que estos costos aumenten, para el principal será menos atractivo ordenar ejecuciones extrajudiciales a sus subalternos. Posteriormente, se realizará el mismo análisis para la función de utilidad del soldado. Para el soldado, la probabilidad de ser castigado por la justicia penal colombiana o ser detectado por la comunidad internacional están relacionados de forma inversa con la cantidad de ejecuciones extrajudiciales que este cometerá. Sin embargo, el aumento en la probabilidad de ser observado por su superior y un incremento en la severidad del castigo estarán relacionados de forma indirecta con el número de falsos positivos que elija el soldado.

Por último, cuando se examina el equilibro se observan ciertas particularidades relacionadas con el efecto que puede tener un parámetro sobre el probable efecto de otro. Dada la naturaleza de este trabajo, se hace necesario analizar algunas particularidades que permitirán dar una visión acerca de qué tan fuerte era la presión del alto mando como determinante del aumento de los falsos positivos y qué tanto podría el castigo penal disminuir su impacto.

\section{Condicionalidades e implicaciones}

Siguiendo la metodología de DeMeritt (2015) se presentarán gráficamente (Figura 2) las predicciones del modelo en función de los valores teóricos de los parámetros. En las ecuaciones de equilibrio (3) y (5) se insertaron valores numéricos y se realizó un cálculo aritmético de las expectativas de los agentes con respecto a la cantidad de falsos positivos ordenada y cometida.

Figura 2. Efectos del aumento de la probabilidad de condena y castigo internacional en falsos positivos ordenados y cometidos dado el monitoreo del alto mando.

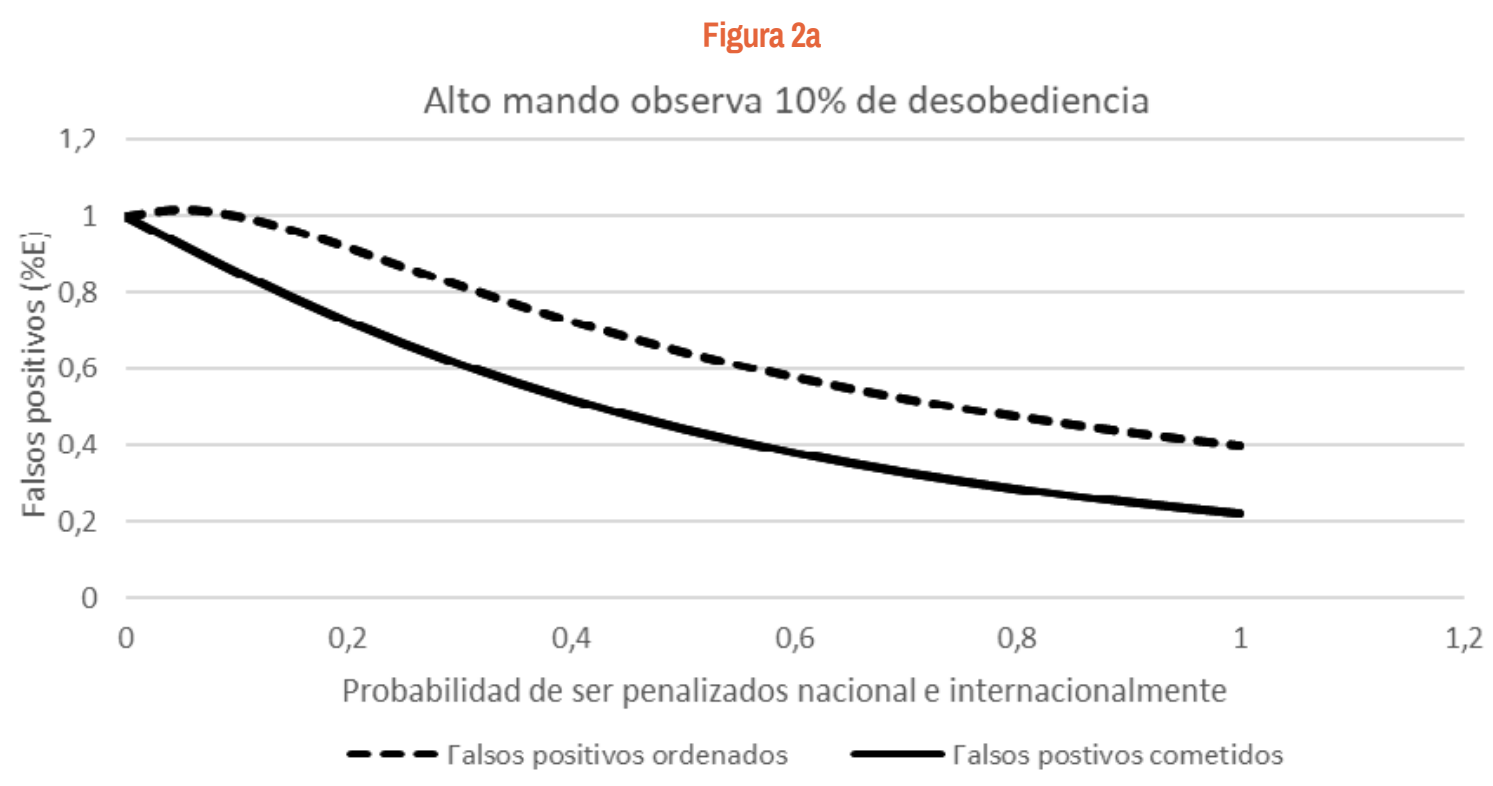


Figura 2b

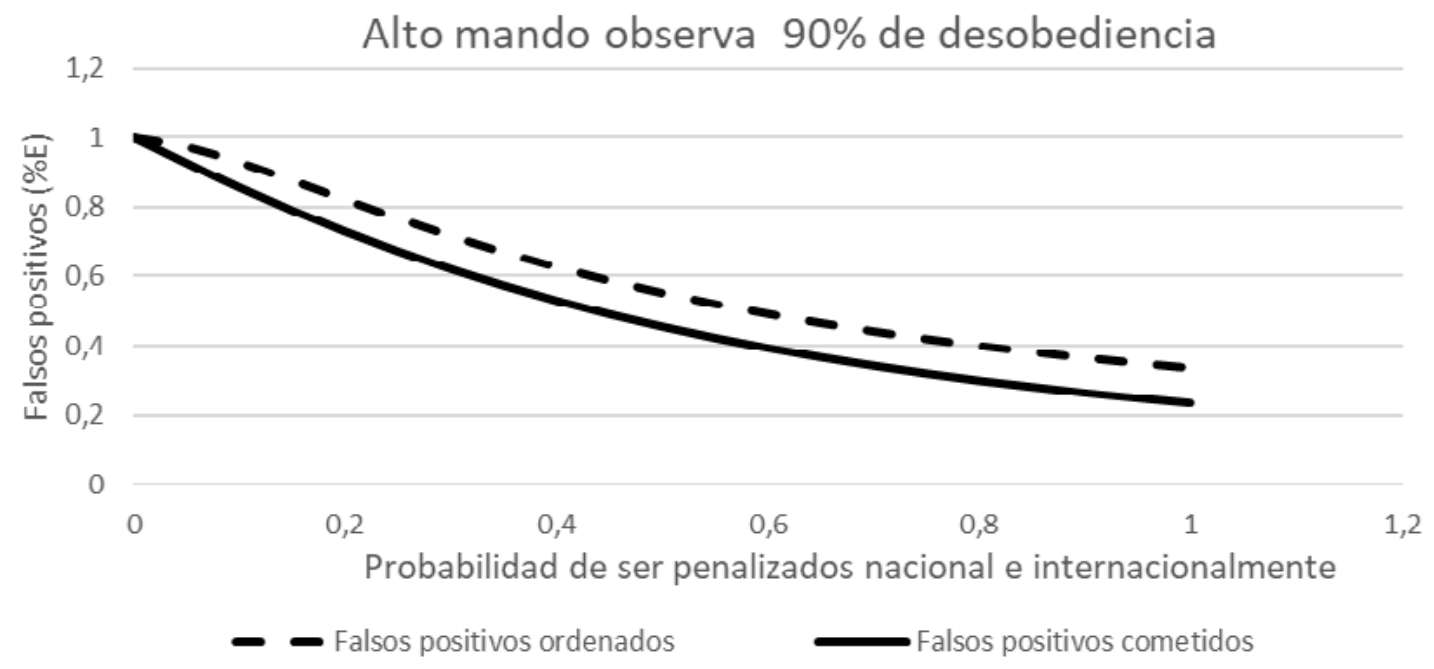

En la Figura 2a se presenta las predicciones sobre cuántas ejecuciones extrajudiciales son ordenadas y cuántas llevadas a cabo por los agentes cuando el alto mando está en la capacidad de observar al $10 \%$ de los soldados desobedientes $(a=0.1)$; así como también la probabilidad esperada de castigo judicial e internacional, tanto para el agente como para el principal, que varía de 0 a 1 , sujeta a que el castigo impuesto por la justicia colombiana al principal es menos probable que para el agente. Todos los demás parámetros se mantendrán constantes en valores medios de sus rangos teóricos. Es posible notar que, aunque la probabilidad de condena reduce la cantidad de ejecuciones extrajudiciales cometidas y ordenadas, dicha reducción no se da en una gran magnitud; esto se debe a que, para el caso de las ejecuciones extrajudiciales en Colombia, los soldados cuentan con un incentivo económico que motiva su comisión, lo cual impide que el número de falsos positivos se acerque a 0 , tan solo por un aumento en la probabilidad de ser condenados por la justicia colombiana.

Ahora, cuando no existe ninguna clase de castigo, el alto mando ordenará exactamente el número de falsos positivos que necesita para cumplir su objetivo y el soldado cumplirá dicha orden al pie de la letra; de esta manera, el número de civiles asesinados y presentados como bajas en combate será el ideal para que el alto mando logre alcanzar el primer puesto en las estadísticas de bajas y reciba beneficios. No obstante, cuando la probabilidad de condena y de castigo internacional es inminente, tanto el alto mando como el soldado van a tratar de evadir dicho costo, razón por la cual los falsos positivos ordenados disminuirán en un $60 \%$ respecto del ideal y los cometidos aproximadamente en un $78 \%$.

La Figura $2 \mathrm{~b}$ muestra la relación entre la probabilidad de condena por asesinato de civiles y el número de ejecuciones extrajudiciales cuando el alto mando observa al $90 \%$ de los soldados desobedientes. Cuando el soldado enfrenta una probabilidad del 0,4 de ser condenado por falsos positivos, pero la probabilidad de ser observado por su superior es tan solo $0,1(a=0,1)$, la diferen- 
cia entre los falsos positivos ordenados y los cometidos es del $43 \%$; mientras que cuando $a=0,9$, dicha diferencia se reduce a un $32 \%$. Este resultado se debe a que el alto mando incurrirá en un costo de castigo si la desobediencia observada aumenta, lo que reducirá el número de ejecuciones extrajudiciales ordenadas. Un aumento en la proporción observada de soldados desobedientes va a aumentar la cantidad de falsos positivos que estos cometen, ya que el agente va a buscar evitar el castigo por desobedecer.

En la Figura 3a mostramos el efecto que tiene el aumento en la probabilidad de condena sobre la capacidad de los altos mandos de obligar a los soldados a cometer falsos positivos usando castigos y represalias. En la Figura 5a, se ve la relación entre un alto mando que utiliza represalias como mecanismo de presión, cuando existe tan solo una probabilidad del $10 \%$ de ser condenado por la justicia colombiana para el soldado y de un $5 \%$ para el alto mando (teniendo en cuenta, que la probabilidad de condena para este es menor que para el soldado). No obstante, la probabilidad de castigo internacional es la misma para el soldado como para el alto mando, por tanto, esta será del $10 \%$ para ambos. Cuando el castigo es nulo, los soldados cometerán aproximadamente un $84 \%$ del número ideal de falsos positivos, mientras que si la violencia del castigo aumenta en un $100 \%$, los soldados solo aumentarán el número de ejecuciones extrajudiciales en un $2 \%$. Dicho aumento no es tan pronunciado debido a que el incentivo económico empujará a los soldados a cometer más falsos positivos cuando observan que las probabilidades de condena y castigo internacional son mínimas, disminuyendo la desviación entre falsos positivos ordenados y cometidos, llevando a que el efecto proporcionado por la violencia del castigo por desobedecer disminuya.

Figura 3. Efectos del aumento en la violencia de la represalia por desobediencia dada la probabilidad de condena por parte de la justicia colombiana y de castigo por parte de la comunidad internacional.

Figura 3a

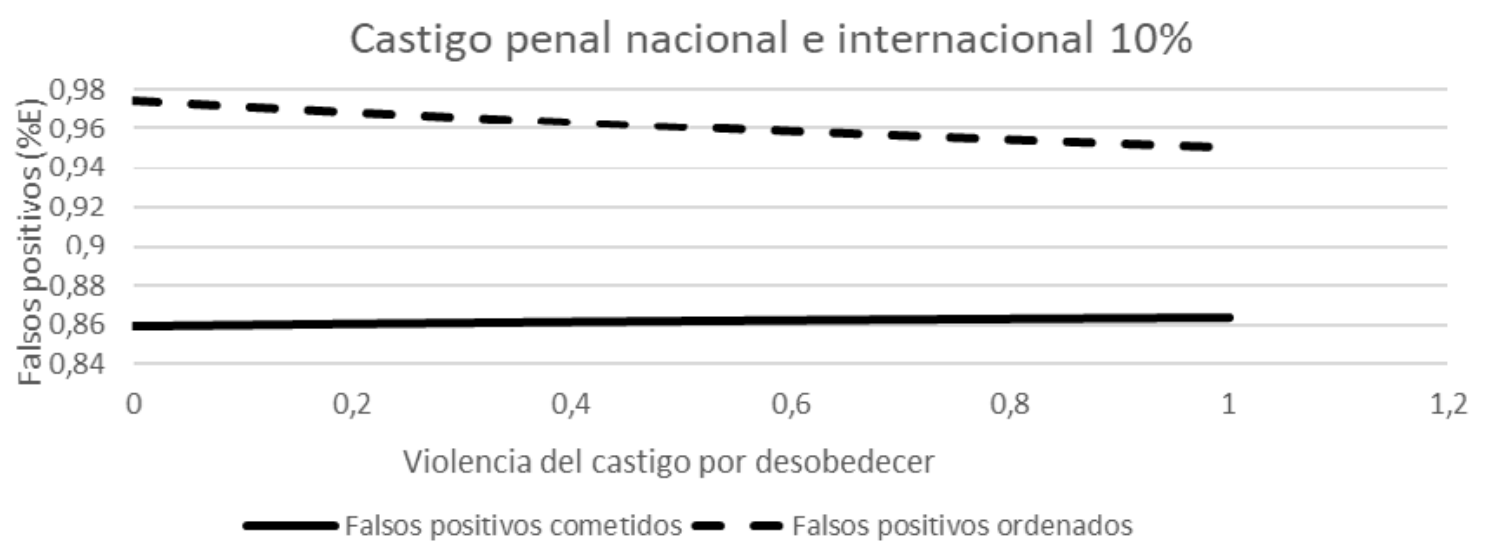




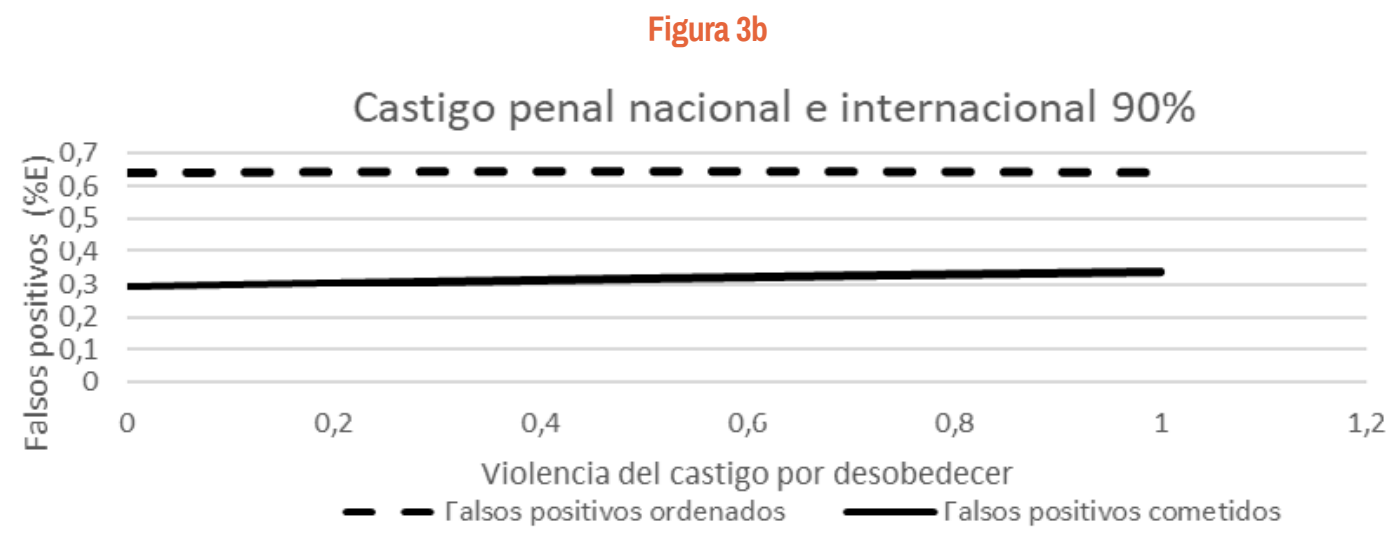

Por otro lado, la Figura $3 \mathrm{~b}$ muestra esta misma relación cuando existe una probabilidad del 90 $\%$ de que el soldado sea condenado por la justicia colombiana y de $45 \%$ para el alto mando, con una probabilidad del $90 \%$ para ambos de ser castigado por la comunidad internacional. En este caso, la cantidad de falsos positivos ordenados disminuye en un $35 \%$ y los cometidos en un 55 $\%$. No obstante, la amenaza de recibir represalias por no cumplir con el número de ejecuciones extrajudiciales ordenadas tiene un mayor efecto en este caso. De esta forma, cuando el alto mando no usa represalias como mecanismo de presión hacia los soldados, estos cometerán aproximadamente un $26 \%$ del conteo de falsos positivos ideales; sin embargo, cuando el alto mando toma represalias y usa la violencia como castigo a la desobediencia, los soldados aumentarán el número de ejecuciones extrajudiciales cometidas en aproximadamente un $6 \%$.

\section{Una justicia imparcial}

Ahora bien, ¿qué sucedería - bajos las condiciones y suposiciones del modelo- si la justicia castigara de igual manera a soldados y superiores? Si los falsos positivos fueran casos aislados y la orden de los altos mandos no fuera motivo suficiente para cometer esta clase de crímenes, la equidad en el castigo para superiores y soldados no debería representar un cambio en el número de ejecuciones extrajudiciales cometidas. Teniendo en cuenta esto, y con el deseo de resolver dicho interrogante, se cambiarán las condiciones del modelo con el fin de comparar dos situaciones: en la primera existirá completa impunidad de los altos mandos, estos solo deberán preocuparse porque la comunidad internacional no logre ver los crímenes que ordenan cometer, mientras que el soldado si correrá con el riesgo de ser condenado por la justicia colombiana por cometer falsos positivos; en la segunda, ambos compartirán la misma probabilidad de ser condenados por la justicia colombiana. La Figura 4 muestra la relación presentada en las Figura 2a y 2b, con la diferencia de que, en este caso, el alto mando gozará de total inmunidad respecto de la justicia nacional en lo que se refiere a falsos positivos. En la Figura 5 se proporcionan los resultados de la misma relación cuando la justicia castiga por igual sin importar el rango. 
Figura 4. Efectos del aumento de la probabilidad de condena y castigo internacional cuando $q_{c}=0$ en falsos positivos ordenados y cometidos, dado el monitoreo del alto mando.

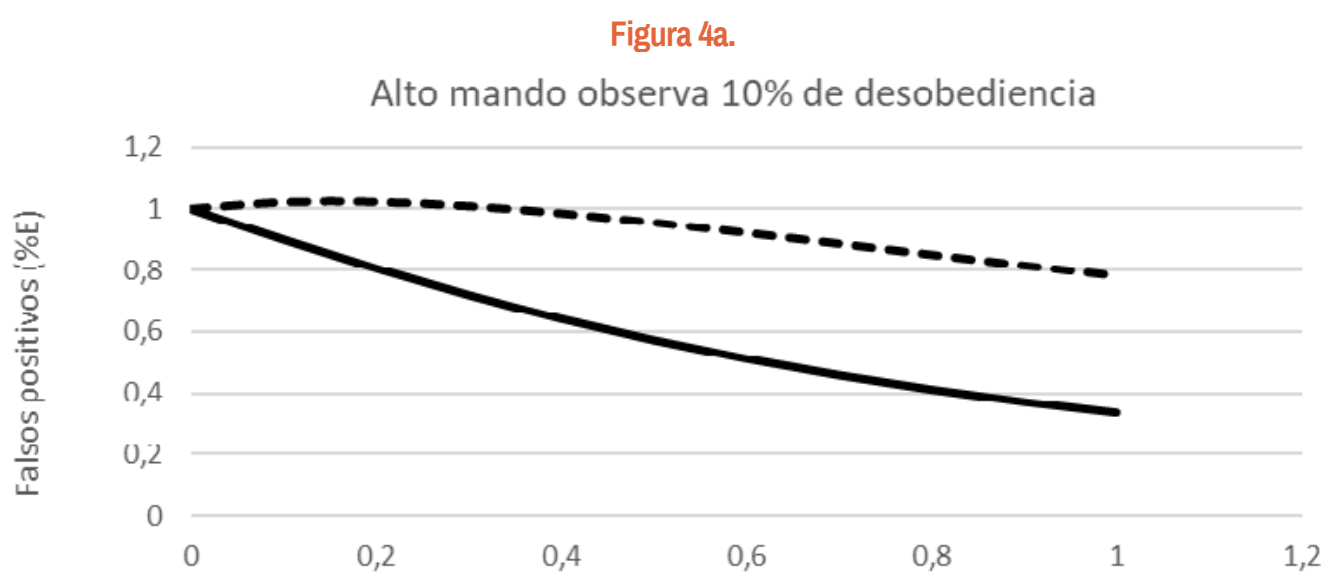

Probabilidad de que el soldado sea penalizado nacional e internacionalmente

- - Falsos positivos ordenados $\quad$ Falsos positivos cometidos

Figura 4b.

Alto mando observa $90 \%$ de desobediencia

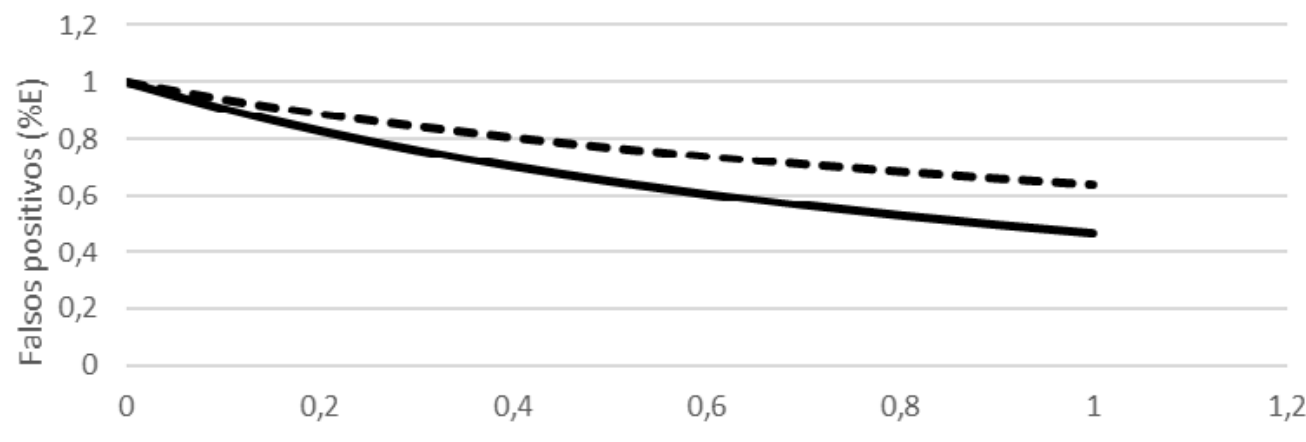

Probabilidad de que el soldado sea penalizado nacional e internacionalmente

_ - Falsos positivos ordenados _ _ Falsos positivos cometidos

Cuando el alto mando observa a un $10 \%$ de los soldados desobedientes y no corre el riesgo de ser condenado por la justicia colombiana, se cometen hasta un $14 \%$ más de ejecuciones extrajudiciales que cuando la justicia es imparcial. De igual forma, cuando $a=0,9$ y existe inmunidad para los altos mandos, se cometen hasta un $26 \%$ más falsos positivos que cuando la justicia castiga por igual al principal y al agente. Cuando el alto mando no percibe la amenaza de ser condenado por estos crímenes, ordena un porcentaje mayor de ejecuciones extrajudiciales y posteriormente el soldado cometerá un mayor número de falsos positivos. Cuando analizamos estas mismas dos situaciones en la relación presentada en la Figura 3, los resultados son similares. La Figura 6 muestra la relación presentada por la Figura 3, con la diferencia de que el único que percibirá la amenaza de ser capturado y condenado por las autoridades colombianas es el soldado; el 
principal, por otro lado, gozará de inmunidad con respecto a las ejecuciones extrajudiciales y tan solo correrá el riesgo de ser observado y castigado por la comunidad internacional. La Figura 7 muestra dicha relación cuando la justicia es imparcial y tanto el principal como el agente perciben la misma amenaza de ser capturados y condenados por cometer falsos positivos.

Figura 5. Efectos del aumento de la probabilidad de condena y castigo internacional cuando la justicia colombiana es imparcial en falsos positivos ordenados y cometidos, dado el monitoreo del alto mando.

Figura 5a

Alto mando observa $90 \%$ de desobediencia

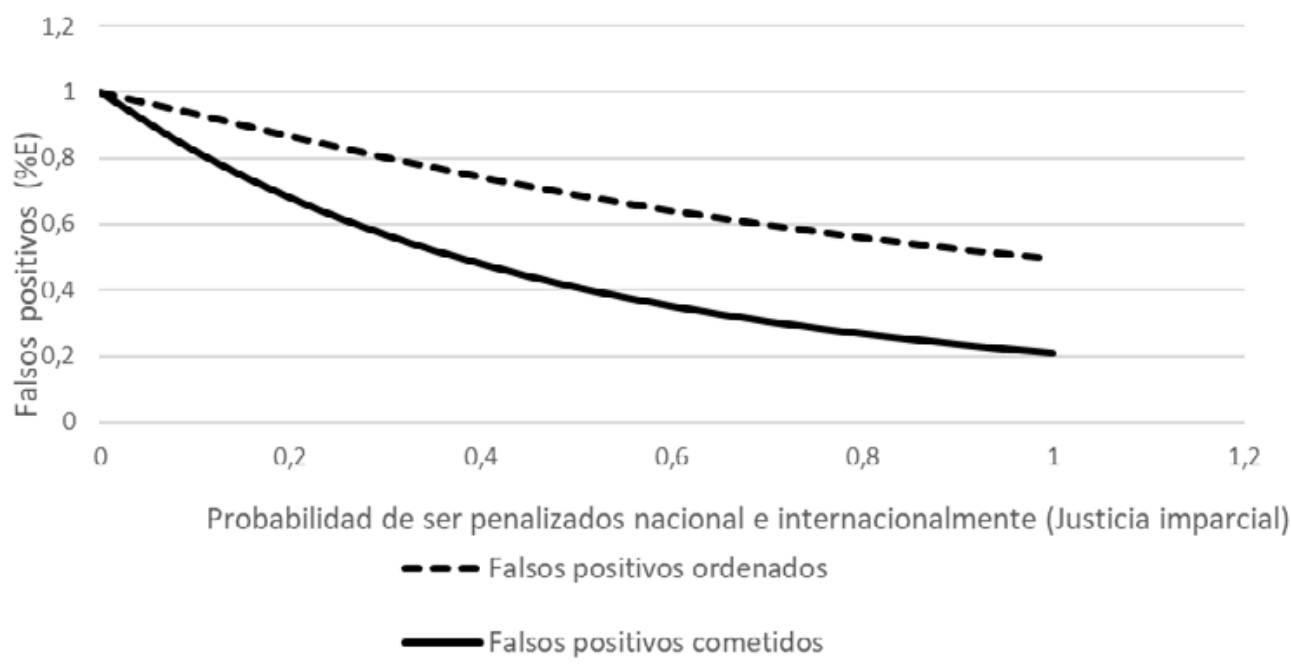

Figura 5b

Alto mando observa $10 \%$ de desobediencia

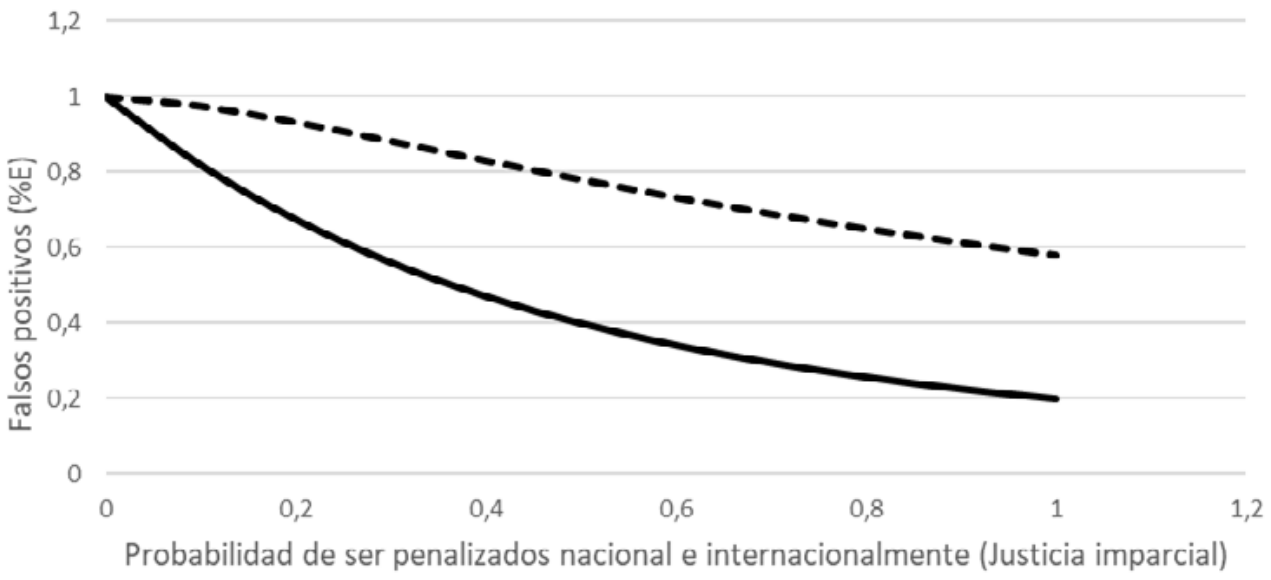

- - Falsos positivos ordenados Falsos positivos cometidos

Fuente: elaboración propia. 
En la Figura 6 se observa que cuando el soldado enfrenta una probabilidad del $10 \%$ de ser condenado por la justicia colombiana y por la comunidad internacional, pero el alto mando sabe que dicha probabilidad para él es nula, este ordenará cometer incluso más falsos positivos de los que necesita para alcanzar su objetivo, y el soldado cometerá aproximadamente un $90 \%$ del ideal de bajas. Además, se cometerán aproximadamente un $14 \%$ más ejecuciones extrajudiciales que cuando la justicia castiga por igual al principal y al agente.

Figura 6. Efectos del aumento en la violencia de la represalia por desobediencia dada la probabilidad de condena por parte de la justicia colombiana y de castigo por parte de la comunidad internacional cuando $q_{c}=0$.
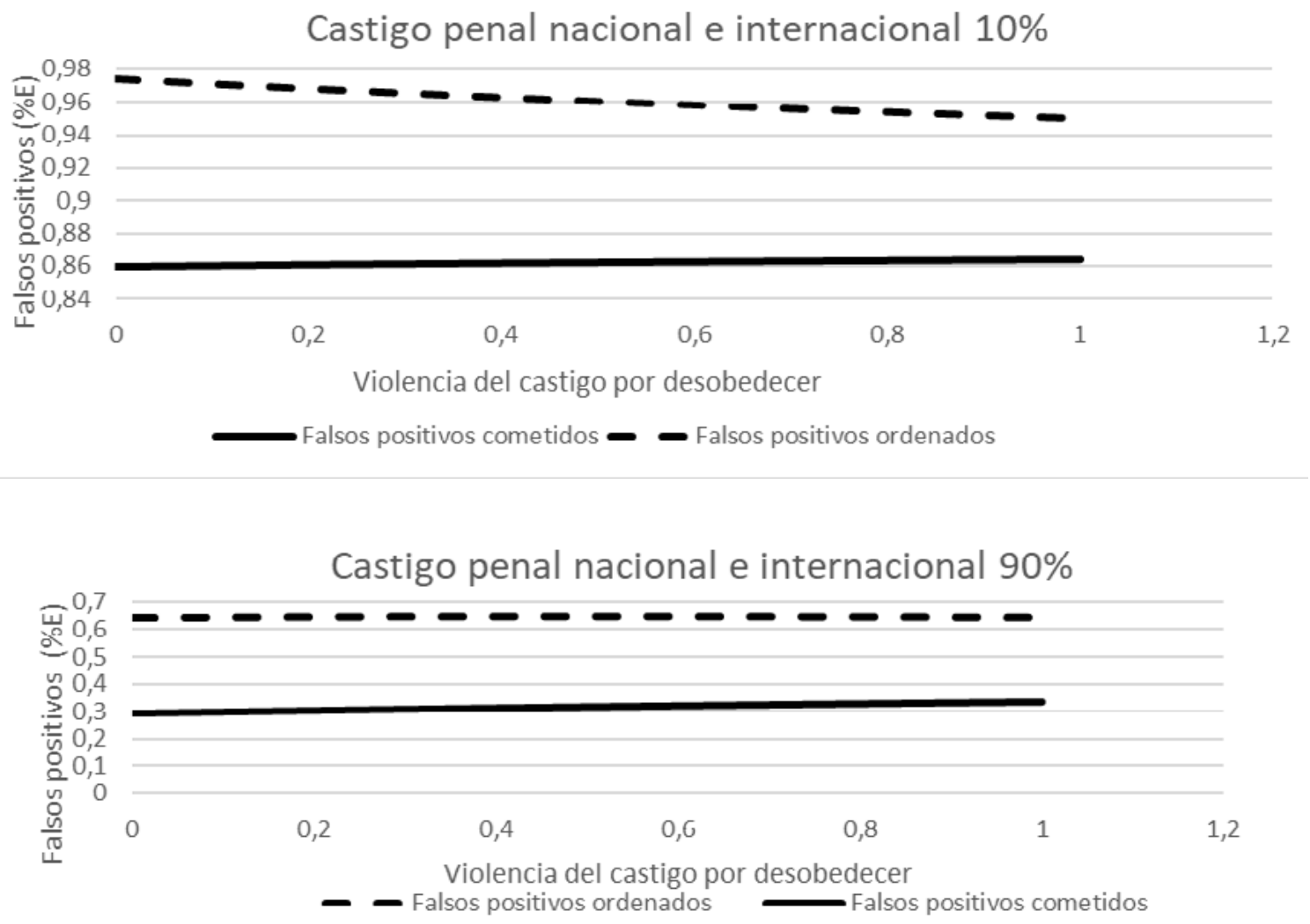

Fuente: elaboración propia.

Cuando el soldado percibe que la probabilidad de ser capturado y condenado asciende a un $90 \%$, evitará dicho castigo cometiendo menos falsos positivos, y el alto mando ordenará menos ejecuciones extrajudiciales; no obstante, cuando la represalia del alto mando aumenta en un $100 \%$ se cometerán aproximadamente un $9 \%$ más de ejecuciones extrajudiciales que cuando la justicia castiga por igual sin importar el rango. 


\section{Conclusión}

Los falsos positivos se extendieron por gran parte del territorio colombiano convirtiéndose en un fenómeno sistematizado en el que varios miembros del Ejército se vieron involucrados. En efecto, nuestros hallazgos revelan que esta clase de crímenes se realizaban con el conocimiento de los altos mandos y, en algunos casos, con su orden y autorización. Por otro lado, la impunidad de la que gozaban los altos mandos del Ejército colombiano facilitó la sistematización de esta clase de crímenes. De la misma forma, aun cuando existe la probabilidad de castigo para el soldado, este se verá obligado a cometer la cuota de falsos positivos incentivado, no solo por las bonificaciones y beneficios, sino por el castigo probable que recibirá del superior.

Este trabajo muestra aspectos importantes antes ignorados cuando se trata del aumento de las ejecuciones extrajudiciales en Colombia durante la implementación de la Política de Seguridad Democrática, entre los que se encuentra el hecho de que los altos mandos fueron responsables de esta clase de delitos, no solo por su indiferencia, sino por la presión por resultados que iba acompañada de represalias a los soldados desobedientes. El hecho de ignorar la responsabilidad de los altos mandos solo perpetua esta clase de crímenes, y al final son los soldados, obligados a obedecer órdenes, a quienes se les condena.

El aumento de las ejecuciones extrajudiciales no es solo resultado de incentivos económicos y falta de monitoreo. El problema tiene bases mucho más profundas relacionadas con una política que medía el éxito de la unidad militar por el número de bajas realizadas. De esta forma, no basta con implementar mecanismos de control y penas más severas para aquellos individuos que cometan esta clase de crímenes, sino que es necesaria una reestructuración de la forma en que se mide el éxito militar.

\section{Conflicto de intereses}

Los autores declaran la inexistencia de conflicto de interés con institución o asociación comercial de cualquier índole. 


\section{Referencias}

Ahnen, R. (2007). Politics of Police Violence in Democratic Brazil. Latin American Politics and Society, 49(1), 141-164. https://doi.org/10.1111/j.1548-2456.2007.tb00377.x

Alston, P. (2010). Report of the Special Rapporteur on Extrajudicial, Summary or Arbitrary Executions. Study on Targeted Killings. United Nations, General Assembly (A/HRC/14/24/ Add. 6).

Arenas, N. (23 de junio de 2015). Así llegaron los "falsos positivos" a los generales. La Silla Vacía. https://lasillavacia.com/historia/asi-llegaron-los-falsos-positivos-los-generales-50609

Butler, C., Tali, G., \& Mitchell, N. (2007). Security Forces and Sexual Violence: A Cross-National Analysis of Principal-Agent Argument. Journal of Peace Research, 44(6), 669-687. https://www.jstor.org/stable/27640594

Caracol Radio. (21 de febrero de 2013). Falsos positivos: impunidad continua. Caracol Radio. http:/caracol.com.co/radio/2013/02/21/judicial/1361457900_846587.html

Cárdenas, E., \& Villa, E. (2013). La política de seguridad democrática y las ejecuciones extrajudiciales. Ensayos Sobre Politica Económica, 31, 64-72. https://doi.org/10.1016/S01204483(13)70011-3

Casey, N. (18 de mayo de 2019). Las órdenes de letalidad del ejército colombiano ponen en riesgo a los civiles, según oficiales. The New York Times. https://www.nytimes.com/ es/2019/05/18/espanol/america-latina/colombia-ejercito-falsos-positivos.html

Centro de Investigación y Educación Popular (CINEP). (2010). Informe especial. 'Falsos positivos', Amenazas colectivas e intolerancia social: balance del segundo semestre de 2009 [En línea]. http://www.cinep.org.co/sites/cinep.cinep.org.co/files/Informe\% $\% 20$ Especial $\% 20$ Cinep-PPP\%20_Falsos\%20Positivos,\%20AC_IS2010[1].pdf

Centro de Investigación y Educación Popular (CINEP). (2011). Deuda con la humanidad II. 23 años de falsos positivos: 1988-2011. CINEP/PPP.

DeMeritt, J. H. (2015). Delegating Death: Military Intervention and Government Killing. Journal of Conflict Resolution, 59(3), 428-454. https://doi.org/10.1177/0022002713515406 
El Espectador. (16 de abril de 2016). El general que frenó los falsos positivos en Sucre. El Espectador. https://www.elespectador.com/noticias/judicial/el-general-freno-los-falsos-positivos-sucre-articulo-627510

El Espectador. (7 de octubre de 2008). Uribe dice que desaparecidos de Soacha murieron en combates. El Espectador. https://www.elespectador.com/noticias/judicial/articulo-uribe-dicedesaparecidos-de-soacha-murieron-combates

Fabre, M.-J. (2015). The Gamification of War in Colombia. Washington Report on the Hemisphere, 35(14), 1.

Feaver, P. D. (1999). Civil-military Relations. Annual Review of Political Science, 2, 211-241. https://doi.org/10.1146/annurev.polisci.2.1.211

Federación Internacional de Derechos Humanos. (2012). Colombia. La guerra se mide en litros de sangre. Falsos positivos, crímenes de lesa humanidad: más altos responsables en la impunidad. https://www.fidh.org/IMG/pdf/colombie589e.pdf

Gómez, S. M. (2011). Constructions of the Violent State: The Case of Extrajudicial Executions of Civilians by State Military Forces in Antioquia, Colombia. Oñati Socio-Legal Series, $1(1), 1-27$.

Humphreys, M., \& Weinstein, J. M. (2006). Handling and Manhandling Civilians in Civil War. American Political Science Research, 100(3), 429-447. https://doi.org/10.1017/ S0003055406062289

Human Rights Watch (2015). El rol de los altos mandos en falsos positivos. https://www.refworld.org.es/docid/577ba56a4.html

Human Rights Watch. (2019). Colombia: Nuevos comandantes del Ejército estarían vinculados con "falsos positivos". Mensaje nocivo para soldados y oficiales, y duro revés para las víctimas. https:/www.hrw.org/es/news/2019/02/27/colombia-nuevos-comandantes-delejercito-estarian-vinculados-con-falsos-positivos.

Jurisdicción Especial para la Paz. (JEP). (18 de febrero de 2021). La JEP hace pública la estrategia de priorización dentro del Caso 03, conocido como el de falsos positivos [Comunicado de prensa]. https://bit.ly/3EhmpkW

Lindsay-Poland, J. (2014). "Guerrillas Killed in Combat" and the Colombian Military's Persistent Impunity. NACLA Report on the Americas, 47(2), 6-10. https://doi.org/10.1080/1071 4839.2014.11721842 
Londoño-Berrío, H. L. (2018). Las funciones políticas de la muerte: ejecuciones extrajudiciales en Colombia, 2002-2010. ABYA-YALA. Revista Sobre Acceso à Justiça e Directos nas Américas, 2(3), 64-100. https://periodicos.unb.br/index.php/abya/article/view/21865/20951

Martín-Córdoba, I. A. (2015). Alcance jurídico de los falsos positivos frente al derecho internacional humanitario, en la justicia ordinaria, la justicia penal militar y la justicia transicional [Ensayo diplomado, Universidad Militar Nueva Granada, Bogotá, Colombia]. Repositorio Institucional UMNG. https://bit.ly/3htACBb

Mitchell, N. J. (2004). Agents of Atrocity: Leaders, Followers, and the Violation of Human Rights in Civil War. Palgrave Macmillan.

Mitchell, N. J. (2012). Democracy's Blameless Leaders: from Dresden to Abu Ghraib, How Leaders Evade Accountability for Abuse, Atrocity, and Killing. NYU Press.

Noticias RCN. (9 de junio del 2014). "Un 20 por ciento de las bajas eran falsos positivos": Del Río. NoticiasRCN.com. https://noticias.canalrcn.com/nacional-justicia/un-20-ciento-lasbajas-eran-falsos-positivos-del-rio

Rodríguez, S. M. (2012). Reporting on Victims of Violence: Press Coverage of the Extrajudicial Killings in Colombia. Signo y Pensamiento, 31(60), 186-213.

Rojas-Bolaños, O. E., \& Benavides-Silva, F. L. (2017). Ejecuciones extrajudiciales en Colombia, 2002-2010. Universidad Santo Tomás.

Schneider, G. (2011). Incentives to Kill: The Organizational Roots of One-Sided Violence. Unpublished Manuscript, University of Konstanz. https://www.researchgate.net/publication/264879325

Schneider, G., Banholzer, L., \& Haer, R. (2011a). Cain's Choice: Causes of One-Sided Violence against Civilians. In Causes of War: An Introduction to Theories behind Warfare and Collective Violence. Nova Science Publishers, 57-82. https://d-nb.info/1099435781/34

Schneider, G., Banholzer, L., \& Albarracin, L. (2011b). Ordered Rape: A Principal-Agent Analysis of Wartime Sexual Violence in the DR Congo. Violence Against Women, 21(11), 13411363. https://doi.org/10.1177/1077801215593645

Semana. (23 de abril del 2015). Falsos positivos una herida que sigue abierta. Semana. https:// www.semana.com/nacion/articulo/verdad-abierta-falsos-positivos-una-herida-que-sigueabierta/425100-3/ 
Semana. (26 de septiembre de 2008). ¿Falsos positivos mortales? Semana. https://www.semana. com/nacion/articulo/falsos-positivos-mortales/95607-3/

Verdad Abierta. (13 de septiembre de 2017). "Las bajas no es lo más importante, es lo único": general Mario Montoya. Verdad Abierta. https://verdadabierta.com/las-bajas-no-es-lo-masimportante-es-lo-unico-general-mario-montoya/

Vivanco, J. M. (19 de marzo de 2018). "En qué parte del mundo quienes cometieron crímenes de guerra están participando en política". Semana. https://www.semana.com/podcast/articulo/semana-en-vivo-entrevista-a-director-de-human-rigths-watch/560870 


\section{Apéndice A}

\section{Demostración de equilibrio}

La utilidad del sodado por cometer un número $v$ de ejecuciones extrajudiciales es:

$$
\begin{gathered}
U s(v)=-a p(f-v)^{2}-i v^{2}-q_{m}(v)^{2}-(f-v)^{2} \\
-a p\left(f^{2}-2 f v+v^{2}\right)-i v^{2}-q_{m}(v)^{2}-\left(f^{2}-2 f v+v^{2}\right) \\
-a p f^{2}+a p 2 f v-a p v^{2}-i v^{2}-q_{m}(v)^{2}-f^{2}+2 f v-v^{2}
\end{gathered}
$$

Realizando la derivada parcial de la utilidad del soldado con respecto a v, y luego maximizando, tenemos:

$$
\begin{gathered}
\frac{\partial U s(v)}{\partial v}=-2\left(\left(q_{m}-a p+i+1\right) v+a f p-f\right) \\
v^{*}=\frac{f(a p+1)}{\left(q_{m}+a p+i+1\right)}
\end{gathered}
$$

Para facilitar la derivación diremos que $z=\frac{a p+1}{q_{m}+a p+i+1}$. Posteriormente, tengamos en cuenta que la función de utilidad del Gobierno por ordenar un número $f$ de falsos positivos es:

$$
\begin{gathered}
U c(f)=-(E-z f)^{2}-i(z f)^{2}-d(f-z f)^{2}-a c(f-z f)^{2}-q_{c}(z f)^{2} \\
U c(f)=-(E-z f)^{2}-i z^{2} f^{2}-(d+a c)(f-z f)^{2}-q_{c} z^{2} f^{2} \\
U c(f)=-(E-z f)^{2}-i z^{2} f^{2}-(d+a c)(1-z)^{2} f^{2}-q_{c} z^{2} f^{2}
\end{gathered}
$$

Realizando la derivada parcial de la utilidad del alto mando con respecto a $f$, y posteriormente maximizando, tenemos:

$$
\begin{gathered}
\frac{\partial U c(f)}{\partial f}=2 z E-2 z^{2} f-2 q_{c} z^{2} f-2 i z^{2} f-2 f(d+a c)(1-z)^{2} \\
=2 z E-2 f\left[z^{2}-q_{c} z^{2}-i z^{2}-(d+a c)(1-z)^{2}\right] \\
f^{*}=\frac{E z}{z^{2}\left(1+q_{c}+i\right)+(d+a c)(1-z)^{2}}
\end{gathered}
$$

Por tanto, existe un único equilibrio perfecto en subjuegos, el cual está dado por:

$$
\left\{v^{*}=\frac{f(a p+1)}{\left(q_{m}+a p+i+1\right)}, f^{*}=\frac{E z}{z^{2}\left(1+q_{c}+i\right)+(d+a c)(1-z)^{2}}\right\}
$$




\section{Apéndice B}

\section{Estática comparativa}

Este modelo tiene un equilibrio único en subjuegos tal que:

$$
\left\{v^{*}=\frac{f(a p+1)}{\left(q_{m}+a p+i+1\right)}, f^{*}=\frac{E z}{z^{2}\left(1+q_{c}+i\right)+(d+a c)(1-z)^{2}}\right\}
$$

Donde, $f^{*}$ es el número óptimo de ejecuciones extrajudiciales que ordena el alto mando al soldado, $v^{*}$ es el número óptimo de falsos positivos que son llevados a cabo después de dicha orden $\mathrm{y} z=\frac{a p+1}{q_{m}+a p+i+1}$. E $>0$ es el porcentaje ideal de falsos positivos; $a \epsilon[0,1]$ es la proporción esperada de desobediencia que el alto mando está en la capacidad de observar, i $\epsilon[0,1]$ es la probabilidad de un castigo internacional por cometer esta clase de crímenes, $q_{m} \epsilon[0,1]$ y $q_{c} \epsilon[0,1]$ son la probabilidad de ser judicializados por las autoridades colombianas del soldado y el alto mando respectivamente. Además, $c>0$ es lo que le cuesta al alto mando castigar la desobediencia, $d \epsilon[0,1]$ es la preocupación del alto mando por proteger su legitimidad y $p>0$ es la violencia esperada por parte del soldado acerca del castigo impuesto por el principal.

Para determinar el efecto de cambiar diferentes parámetros en el resultado de equilibrio, se realizan segundas derivadas parciales de la utilidad del alto mando y del soldado con respecto a cada influencia exógena. El signo de esta segunda derivada revelará el efecto direccional de aumentar cada paramento en la variable dependiente a estudiar. Para empezar, se realizará el análisis de estática comparativa para la cantidad de falsos positivos ordenada:

$$
f^{*}=\frac{E z}{z^{2}\left(1+q_{c}+i\right)+(d+a c)(1-z)^{2}}
$$

Para determinar el efecto que tiene un aumento del costo de castigar la desobediencia sobre el número de falsos positivos ordenado, se calcula $\frac{\partial^{2} U c(f)}{\partial c}$ :

$$
\begin{gathered}
\frac{\partial U c(f)}{\partial f}=2 z E-2 z^{2} f-2 q_{c} z^{2} f-2 i z^{2} f-2 f(d+a c)(1-z)^{2} \\
=2 z E-2 f\left[z^{2}-q_{c} z^{2}-i z^{2}-(d+a c)(1-z)^{2}\right] \\
f^{*}=\frac{E z}{z^{2}\left(1+q_{c}+i\right)+(d+a c)(1-z)^{2}} \\
\frac{\partial^{2} U c(f)}{\partial c}=-\frac{E a(1-z)^{2}}{\left((1-z)^{2}(a c+d)+\left(q_{c}+i+1\right) z^{2}\right)^{2}}
\end{gathered}
$$


$\frac{\partial^{2} U c(f)}{\partial c}$ es estrictamente negativa. Por tanto, a medida que se castiga la desobediencia se hace más costoso y el alto mando ordenará disminuir el número de falsos positivos cometidos.

Por otro lado, para determinar el efecto de un aumento de la preocupación del alto mando por su legitimidad sobre el número de ejecuciones extrajudiciales ordenadas, se calcula $\frac{\partial^{2} U c(f)}{\partial d}$ :

$$
\frac{\partial^{2} U c(f)}{\partial d}=-\frac{E z(1-z)^{2}}{\left((1-z)^{2}(a c+d)+\left(q_{c}+i+1\right) z^{2}\right)^{2}}
$$

$\frac{\partial^{2} U c(f)}{\partial d}$ es estrictamente negativa. Esto implica que, a medida que el alto mando aumenta su preocupación por su propia legitimidad, ordenará cometer un menor número de ejecuciones extrajudiciales.

Posteriormente, se va a realizar el análisis de estática comparativa para falsos positivos que el soldado efectivamente lleva a cabo.

$$
v^{*}=\frac{f(a p+1)}{\left(q_{m}+a p+i+1\right)}
$$

Para terminar el efecto sobre el número de falsos positivos cometidos de un incremento la proporción esperada de desobediencia observada se calcula $\frac{\partial^{2} U s(v)}{\partial a}$ :

$$
\frac{\partial^{2} U s(v)}{\partial a}=\frac{f p\left(q_{m}+i\right)}{\left(p a+q_{m}+i+1\right)^{2}}
$$

$\frac{\partial^{2} U s(f)}{\partial a}$ es estrictamente positiva. Por tanto, a medida que el alto mando observa una mayor proporción de soldados desobedientes, el número de falsos positivos cometidos aumenta.

Posteriormente, para determinar el efecto de un incremento del castigo internacional esperado por cometer falsos positivos sobre el número cometido de estos, calculamos $\frac{\partial^{2} U s(v)}{\partial i}$ :

$$
\frac{\partial^{2} U s(v)}{\partial i}=-\frac{f(a p+1)}{\left(i+q_{m}+a p+1\right)^{2}}
$$

$\frac{\partial^{2} U s(v)}{\partial i}$ es estrictamente negativa. Esto quiere decir que a medida que la probabilidad de castigo internacional aumenta, los soldados cometerán un número menor de falsos positivos.

De igual forma, para calcular el impacto de un aumento en la probabilidad de castigo judicial por parte de la justicia colombiana sobre el número de falsos positivos cometidos calcularemos $\frac{\partial^{2} U s(v)}{\partial q_{m}}$ :

$$
\frac{\partial^{2} U s(v)}{\partial q_{m}}=-\frac{f(a p+1)}{\left(i+q_{m}+a p+1\right)^{2}}
$$


$\frac{\partial^{2} U s(v)}{\partial q_{m}}$ es estrictamente negativo, por lo que un incremento en la probabilidad de castigo judicial por parte de la justicia Colombia trae consigo una disminución de los falsos positivos llevados a cabo por los soldados.

Por último, para determinar el efecto que tiene un incremento en la violencia del castigo sobre el número de falsos positivos que se llevarán a cabo se calcula $\frac{\partial^{2} U s(v)}{\partial p}$ :

$$
\frac{\partial^{2} U s(v)}{\partial p}=\frac{a f\left(q_{m}+i\right)}{\left(i+q_{m}+a p+1\right)^{2}}
$$

$\frac{\partial^{2} U s(v)}{\partial p}$ es estrictamente positiva, por tanto, un incremento en la violencia del castigo por parte del alto mando provocará aumento en los falsos positivos llevados a cabo por los soldados. 